\title{
Adherence to prenatal iron-folic acid supplementation in low- and middle- income countries (LMIC): a protocol for systematic review and meta-analysis
}

Mohammed Akibu ${ }^{1,7^{*}+}$, Tesfalidet Tekelab ${ }^{2,3}$, Abdella Amano ${ }^{4}$, Merga Besho ${ }^{3}$, Stephanie Grutzmacher ${ }^{5}$, Mesfin Tadese ${ }^{1}$ and Tesfa Dejenie Habtewold ${ }^{6+}$ (D)

\begin{abstract}
Background: Daily iron-folic acid supplementation reduces anemia and various adverse obstetric outcomes such as preterm delivery, low birthweight, hemorrhage, and perinatal and maternal morbidity and mortality. However, its supplementation has not been successful that attributed to several determinants including poor adherence. Therefore, we aimed to conduct a systematic review and meta-analysis on the prevalence and determinants of adherence to prenatal iron-folic acid supplementation in low- and middle-income countries. In addition, we will develop a conceptual framework in the context of low- and middle-income countries (LMIC).

Methods/design: We will search PubMed, MEDLINE, EMBASE, EBSCO, Web of Science, SCOPUS, WHO Global Index Medicus, and African Journals Online (AJOL) databases to retrieve relevant literatures. Observational (i.e., casecontrol, cohort, cross-sectional, survey, and surveillance reports) and quasi-randomized and randomized controlled trial studies conducted in LMIC will be included. The Newcastle-Ottawa Scale (NOS) and Joanna Briggs Institute (JBI) critical appraisal checklist will be used to assess the quality of observational and randomized controlled trial studies respectively. The pooled prevalence and odds ratio of determinants of adherence will be generated using a weighted inverse-variance meta-analysis model. Statistical heterogeneity among studies will be assessed by Cochran's $Q X^{2}$ statistics and Higgins ( $I^{2}$ statistics) method. The result will be presented using forest plots and Harvest plots when necessary. Furthermore, we will perform Jackknife sensitivity and subgroup analysis. Data will be analyzed using comprehensive meta-analysis software (version 2).

Discussion: Contemporary evidence about the prevalence and determinants of adherence in LMIC will be synthesized to generate up-to-date knowledge. To our knowledge, this is the first systematic review. It would have substantial implications for researchers, clinicians, and policymakers for optimizing maternal and child health outcomes in LMIC.

\footnotetext{
* Correspondence: mahammedakibu@gmail.com

${ }^{\dagger}$ Mohammed Akibu and Tesfa Dejenie Habtewold contributed equally to this work.

'Institute of Medicine and Health Science, Debre Berhan University, Debre

Berhan, Ethiopia

${ }^{7}$ Department of Midwifery, Institute of Medicine and Health Science, Debre

Berhan University, P.O. Box 445, Debre Berhan, Ethiopia

Full list of author information is available at the end of the article
}

(c) The Author(s). 2018 Open Access This article is distributed under the terms of the Creative Commons Attribution 4.0 International License (http://creativecommons.org/licenses/by/4.0/), which permits unrestricted use, distribution, and reproduction in any medium, provided you give appropriate credit to the original author(s) and the source, provide a link to the Creative Commons license, and indicate if changes were made. The Creative Commons Public Domain Dedication waiver (http://creativecommons.org/publicdomain/zero/1.0/) applies to the data made available in this article, unless otherwise stated. 
(Continued from previous page)

Systematic review registration: The protocol has been registered on International Prospective Register of Systematic Review (PROSPERO), University of York Center for Reviews and Dissemination (https://www.crd.york.ac.uk), registration number CRD42017080245.

Keywords: Folic acid, Folate, Iron, Medication adherence, Patient compliance, Prenatal Nutritional Physiological Phenomena, Prenatal care, Pregnancy

\section{Background}

Anemia is a global public health problem affecting nearly two billion people [1]. Despite vulnerability across the population, anemia is prevalent in pregnant woman (> $40 \%$ ) and young children because of increased demand and iron-folic acid deficiency [2]. Iron-folic acid deficiency increases the risk of blood loss during labor, maternal mortality, preterm delivery, low birthweight, and perinatal mortality [3, 4]. Thus, to prevent these poor health outcomes, the World Health Organization (WHO) has recommended that all pregnant women take a standard dose of $60 \mathrm{mg}$ of elemental iron along with $400 \mu \mathrm{g}$ of folic acid daily for the first 6 months. Additionally, in areas where the prevalence of anemia is over $40 \%$, the WHO recommends postpartum supplementation for 3 months [2].

A systematic review and meta-analysis of maternal anemia in lower and middle-income countries (LMIC) showed that 12,19 , and $18 \%$ of low birthweight, preterm births, and perinatal mortality are associated with maternal anemia respectively [5]. Accumulated body of evidence shows that prenatal iron-folic acid supplementation reduces maternal anemia and associated adverse perinatal outcomes, such as low birthweight, and maternal and newborn mortality $[6,7]$.

In LMIC, high proportion of pregnant women suffered from anemia mainly due to poor adherence to the daily iron-folic acid regimen $[1,8]$. Furthermore, various individual studies shows that poor adherence is common in LMIC [9-11]. In our review, adherence is defined as taking at least five iron-folic acid tablets per week [2] or percentage of women who consumed more than $70 \%$ of the recommended daily dose [12].

Generally, pregnant women living in LMIC need a support for initiating and maintaining optimal adherence to the recommended iron-folic acid supplementation [13]. Therefore, this systematic review and meta-analysis aimed to (1) investigate the level of adherence to prenatal iron-folic acid supplementation, (2) identify its determinants, and (3) develop PRECEDE-PROCEED adherence conceptual framework in the context of LMIC.

\section{Methods/design}

\section{Reporting of the review findings}

This protocol has been written in accordance with the recommendation of Preferred Reporting Items for
Systematic Review and Meta-Analysis (PRISMA-P) 2015 statement [14]. The PRISMA-P Elaboration and Explanation document is also used to develop the protocol [15] (Additional file 1). The protocol has been registered on International Prospective Register of Systematic Review (PROSPERO), University of York Center for Reviews and Dissemination [https://www.crd.york.ac.uk/], registration number CRD42017080245. Preferred Reporting Items for Systematic review (PRISMA-2009) statement will be used to report the findings. Furthermore, PRISMA flow diagram will be used to illustrate study screening and selection process.

\section{PECO search guide}

- P-population: pregnant mothers who have received prenatal iron-folic acid

- E-exposure: determinants of adherence (e.g., income level, educational status) and interventions and intervention characteristics/components (e.g., how the supplements are delivered to the women, whether they receive instruction on how and why they should take them, reminders that are sent via text message) that are associated with prenatal ironfolic adherence. PRECEDE-PROCEED conceptual framework [10] will be used to select potential exposures/determinants

- C-comparison: the reported reference group for each determinant in each study (e.g., adherence in pregnant women with high educational status versus adherence in women with low educational status)

- O-outcome: adherence to iron-folic acid supplementation

\section{Data source and search strategy}

PubMed, MEDLINE, EMBASE, EBSCO, Web of Science, and SCOPUS databases will be searched to retrieve all available studies. We will also extend our searching to WHO Global Index Medicus and African Journals Online (AJOL). Cross-references of included studies will be hand-searched as well to access additional relevant articles that may have been missed in the search. In addition, we will search existing reviews and perform citing studies/snowballing search in PubMed and SCOPUS databases to screen all studies that cited included 
studies. Likewise, a search for gray literature will be conducted using Google Scholar and through browsing Hinari (http://www.who.int/hinari/en/), Carolina digital repository (https://cdr.lib.unc.edu/), and SpringerOpen-Open repository (https://www.springeropen.com/get-published/ indexing-archiving-and-access-to-data/open-repository).

Medical subject headings (Mesh), keywords, and free-text words were identified for selected PECO components. "OR", "AND", and "NOT" Boolean operators were used to combine search terms. Moreover, we will contact Cochrane Pregnancy and Childbirth Group, WHO headquarters and regional offices, the nutrition section of the United Nations Children's Fund (UNICEF), the World Food Program (WFP), and US Agency for International Development (USAID) micronutrient program to identify additional studies [16]. The search strategy for PubMed database has been designed in consultation with medical information specialist and supplemented with this protocol (Additional file 2). The Peer Review of Electronic Search Strategies (PRESS) 2015 guideline statement is followed to prepare the search strategy [17].

\section{Eligibility criteria}

The inclusion criteria are (1) observational (i.e., case-control, cohort, cross-sectional, survey, and surveillance reports) and quasi-randomized and randomized controlled trial studies, (2) studies conducted in LMIC, and (3) studies that reported the prevalence and/or least adjusted determinants of adherence. The most up-to-date World Bank country classification, when our review is published, will be used to categorize LMIC [18]. The search will not be restricted to any language and publication year. Qualitative studies that thematically analyzed the determinants of adherence will be included. Studies conducted in study populations other than pregnant women will be excluded. Moreover, case reports and expert opinion will be excluded. We will pilot the eligibility criteria in at least 200 references and double-check if they allow unambiguously included or excluded studies.

\section{Selection of studies}

Covidence web-based software will be used to remove duplicated articles and carry out all of the screening processes. First, articles will be assessed for inclusion through a title and abstract review by two independent reviewers. Disagreement will be solved by consensus; a third reviewer will be invited in case of persistent contradiction. Second, potentially eligible studies will undergo full-text review to determine if they satisfy the criteria set for inclusion. We will do a full-text review in duplicates and clearly document reasons for inclusion and exclusion. Finally, data will be extracted from all articles that meet the inclusion criteria.

\section{Data extraction}

Data will be extracted using the Joanna Briggs Institute (JBI) data extraction form for experimental/observational studies (Additional file 3) [19]. The data extraction form will be pre-tested with 3-5 eligible studies. Two reviewers will independently extract all relevant information including study setting, sample size, prevalence of adherence to iron-folic acid supplementation, least-adjusted determinants, and source of funding. The prevalence of adherence to iron-folic acid will be extracted only if reported and/or estimated based on experts' opinion or previously published studies or guidelines. In case of incomplete data, the corresponding author(s) will be contacted to find full information. Disagreement between reviewers will be resolved by consensus.

\section{Quality assessment}

The quality of all included studies will be rigorously assessed by two independent reviewers. The Newcastle-Ottawa Scale (NOS) will be used to assess the quality of cohort and case-control articles [20]. Similarly, cross-sectional studies will be examined using NOS adapted for cross-sectional studies. NOS has a good inter-rater reliability and validity [21]. The NOS criteria and its rating system have been published elsewhere [22]. The Joanna Briggs Institute (JBI) critical appraisal checklist will be used to assess the quality of quasi-randomized controlled trials [23].

\section{Data synthesis and analysis}

Data will be analyzed using comprehensive meta-analysis software (version 2) [24]. Funnel plots and Egger's regression test will be used to examine the possible risk of publication bias. Heterogeneity among studies will be assessed by Cochran's $Q X^{2}$ statistics and Higgins ( $I^{2}$ statistics) method [25]. $I^{2}$ describes the percentage of total variations across the studies due to heterogeneity rather than chance. $I^{2}$ value greater than $80 \%$ will be indicative of considerable heterogeneity [26]. In addition, the heterogeneity among studies will be checked manually in terms of study population, geographic distribution, and methods. The pooled prevalence and odds ratio (OR) of determinants of adherence will be generated using a weighted inverse-variance meta-analysis model. If substantial heterogeneity is detected, random-effects model results will be reported. Arcsine transformation will be carried out to minimize the effect of studies with very high or low effect size if normality assumption will be fulfilled [27]. The result will be presented using forest plots [28] and Harvest plots [29] when necessary. Qualitative analysis will be performed to construct a PRECEDE-PROCEED conceptual framework in the context of LMIC [10]. PRECEDE-PROCEED conceptual framework includes four groups of factors: predisposing factors (e.g., age, educational status, income, knowledge on anemia and prevention), enabling factors 
(e.g., number of iron/folate tablets received, acceptability of iron/folate supplements, sides effects), reinforcing factors (e.g., number of antenatal care visits, use of reminding techniques, support from family and relatives), and environmental factors (e.g., availability of antenatal care, access to antenatal care) [10].

\section{Sensitivity analysis}

Leave-one-out Jackknife sensitivity analysis will be used to stabilize the variance of studies with very low or very large prevalence estimates [30,31]. If the point estimate of the new pooled effect size is outside of the $95 \%$ confidence interval of the original/previous pooled effect size, it will be concluded that the excluded study has a significant effect on the pooled estimate. Thus, the study should not be included in the final analysis whether the effect of the study is too small or too large. In addition, the randomand fixed-effects model will be compared and decision will be made based on the best fitting model to the data.

\section{Subgroup analysis}

Subgroup analysis will be carried out based on epidemiological and/or clinical covariates that can impact estimates such as study design, measures of adherence, age of women, residence of women, and geographical distribution.

\section{Potential methodological amendments}

If protocol modifications are required, we will include the detailed description of any changes along with a justification during the publication of the review results.

\section{Discussion}

The protocol has been registered and written in accordance with a standardized guideline which is helpful to replicate methods in other nations. Contemporary evidence on the prevalence and determinants of adherence in LMIC will be synthesized using an appropriate statistical method and qualitatively using PRECEDE-PROCEED conceptual framework [10]. Given that this is the first study, it would have substantial implications for researchers, clinicians, and policymakers in LMIC. We will develop a conceptual framework in the context of LMIC which could be helpful for prioritizing problems in primary healthcare and building statistical models for researchers in LMIC.

\section{Abbreviations}

JBI: Joanna Briggs Institute; LMIC: Low- and middle-income countries; NOS: Newcastle-Ottawa Scale; PECO: Population, exposure, comparator, and outcome; PROSPERO: Prospective Register of Systematic Review; RCTs: Randomized controlled trials

\section{Acknowledgements}

We would like to forward our heartfelt gratitude to Sjoukje van der Werf (medical information specialist at University of Groningen, The Netherlands) for her generous support during the development of search strings.

\section{Authors' contributions}

MA conceived and designed the study. TD developed the search strategy. MA, TT, TD, MB, MT, SG and AA wrote and prepared the protocol. TD, SG and MA revised the protocol. All authors read and approved the final protocol.

\section{Competing interest}

The authors declare that they have no competing interests.

Ethics approval and consent to participate

Not applicable

Consent for publication

Not applicable

\section{Publisher's Note}

Springer Nature remains neutral with regard to jurisdictional claims in published maps and institutional affiliations.

\section{Author details}

${ }^{1}$ Institute of Medicine and Health Science, Debre Berhan University, Debre Berhan, Ethiopia. ${ }^{2}$ Research Centre for Generational Health and Ageing at the Hunter Medical Research Institute, University of Newcastle, Callaghan Australia. ${ }^{3}$ College of Medicine and Health Science, Wollaga University, Nekemte, Ethiopia. ${ }^{4}$ College of Medicine and Health Science, School of Public Health, Hawassa University, Hawassa, Ethiopia. ${ }^{5}$ School of Biological and Population Health Sciences, Oregon State University, Corvallis, USA. ${ }^{6}$ Department of Epidemiology, University Medical Center Groningen, University of Groningen, Groningen, The Netherlands. 'Department of Midwifery, Institute of Medicine and Health Science, Debre Berhan University, P.O. Box 445, Debre Berhan, Ethiopia.

Received: 26 March 2018 Accepted: 11 July 2018

Published online: 25 July 2018

\section{Additional files}

Additional file 1: PRISMA-P (Preferred Reporting Items for Systematic review and Meta-Analysis Protocols) 2015 checklist: recommended items to address in a systematic review protocol*. (DOCX $22 \mathrm{~kb}$ )Additional file 2: PubMed search string. (DOCX $16 \mathrm{~kb}$ )Additional file 3: JBI Data Extraction Form for Experimental/Observational Studies. (DOCX $80 \mathrm{~kb}$ )

\section{Author details}

${ }^{1}$ Institute of Medicine and Health Science, Debre Berhan University, Debre Berhan, Ethiopia. ${ }^{2}$ Research Centre for Generational Health and Ageing at the Hunter Medical Research Institute, University of Newcastle, Callaghan, Australia. ${ }^{3}$ College of Medicine and Health Science, Wollaga University, Nekemte, Ethiopia. ${ }^{4}$ College of Medicine and Health Science, School of Public Health, Hawassa University, Hawassa, Ethiopia. ${ }^{5}$ School of Biological and Population Health Sciences, Oregon State University, Corvallis, USA ${ }^{6}$ Department of Epidemiology, University Medical Center Groningen, University of Groningen, Groningen, The Netherlands. 'Department of Midwifery, Institute of Medicine and Health Science, Debre Berhan University, P.O. Box 445, Debre Berhan, Ethiopia.

Received: 26 March 2018 Accepted: 11 July 2018

Published online: 25 July 2018

\section{References}

1. Bailey RL, West KP Jr, Black RE. The epidemiology of global micronutrient deficiencies. Ann Nutr Metab. 2015;66(Suppl 2):22-33.

2. World Health Organization. Daily iron and folic acid supplementation during pregnancy. WHO (cited 24 Jan 2016). Available from URL: http://www.who. int/elena/titles/guidance summaries/daily iron pregnancy/en.

3. Pasricha SR, Drakesmith $\bar{H}$, Black J, Hipgrave D, Biggs BA. Control of iron deficiency anemia in low- and middle-income countries. Blood. 2013;121: 2607-17. 
4. Kapil U, Singh Bhadoria A. National iron-plus initiative guidelines for control of iron deficiency anaemia in India, 2013. 2014.

5. Rahman MM, Abe SK, Rahman MS, Kanda M, Narita S, Bilano V, et al. Maternal anemia and risk of adverse birth and health outcomes in low-and middle-income countries: systematic review and meta-analysis, 2. Am J Clin Nutr. 2016;103:495-504.

6. Haider BA, Olofin I, Wang M, Spiegelman D, Ezzati M, Fawzi WW, et al. Anaemia, prenatal iron use, and risk of adverse pregnancy outcomes: systematic review and meta-analysis. BMJ. 2013;346:f3443.

7. Pena-Rosas JP, Viteri FE. Effects and safety of preventive oral iron or iron +folic acid supplementation for women during pregnancy. Cochrane Database Syst Rev. 2009;(4):CD004736.

8. Black RE, Victora CG, Walker SP, Bhutta ZA, Christian P, De Onis M, et al. Maternal and child undernutrition and overweight in low-income and middle-income countries. Lancet. 2013;382:427-51.

9. Arega Sadore A, Abebe Gebretsadik L, Aman HM. Compliance with ironfolate supplement and associated factors among antenatal care attendant mothers in Misha district, South Ethiopia: community based cross-sectional study. J Environ Public Health. 2015;2015

10. Lacerte $P$, Pradipasen $M$, Temcharoen $P$, Imamee $N$, Vorapongsathorn $T$. Determinants of adherence to iron/folate supplementation during pregnancy in two provinces in Cambodia. Asia Pacific Journal of Public Health. 2011:23:315-23.

11. Ogundipe O, Hoyo C, Østbye T, Oneko O, Manongi R, Lie RT, et al. Factors associated with prenatal folic acid and iron supplementation among 21,889 pregnant women in Northern Tanzania: a cross-sectional hospital-based study. BMC Public Health. 2012;12:481.

12. Low MSY, Speedy J, Styles CE, De-Regil LM, Pasricha S. Daily iron supplementation for improving anaemia, iron status and health in menstruating women. The Cochrane Library. 2016.

13. Ladipo OA. Nutrition in pregnancy: mineral and vitamin supplements. Am J Clin Nutr. 2000;72:280S-90S.

14. Moher D, Shamseer L, Clarke M, Ghersi D, Liberati A, Petticrew M, et al. Preferred reporting items for systematic review and meta-analysis protocols (PRISMA-P) 2015 statement. Systematic reviews. 2015;4:1.

15. Shamseer L, Moher D, Clarke M, Ghersi D, Liberati A, Petticrew M, et al. Preferred reporting items for systematic review and meta-analysis protocols (PRISMA-P) 2015: elaboration and explanation. BMJ. 2015;350:g7647.

16. De-Regil LM, Peña-Rosas JP, Fernández-Gaxiola AC, Rayco-Solon P. Effects and safety of periconceptional oral folate supplementation for preventing birth defects. The Cochrane Library. 2015.

17. McGowan J, Sampson M, Salzwedel DM, Cogo E, Foerster V, Lefebvre C. PRESS peer review of electronic search strategies: 2015 guideline statement. J Clin Epidemiol. 2016;75:40-6.

18. Bank W. Country and lending groups. World Bank 2015.

19. Munn Z, Tufanaru C, Aromataris E. JBI's systematic reviews: data extraction and synthesis. Am J Nurs. 2014;114:49-54.

20. Wells G, Shea B, O'connell D, Peterson J, Welch V, Losos M, et al. The Newcastle-Ottawa Scale (NOS) for assessing the quality of nonrandomised studies in meta-analyses. ohri.ca/programs/clinical_epidemiology/oxford.asp (accessed June 2014). 2008

21. Hartling L, Hamm M, Milne A, Vandermeer B, Santaguida PL, Ansari M, et al. Validity and inter-rater reliability testing of quality assessment instruments [Internet]. 2012;12-EHC039-EF.

22. McPheeters ML, Kripalani S, Peterson NB, Idowu RT, Jerome RN, Potter SA et al. Closing the quality gap: revisiting the state of the science (vol. 3: quality improvement interventions to address health disparities). Evid Rep Technol Assess (Full Rep). 2012;(208.3):1-475.

23. The Joanna Briggs Institute (JBI). Checklist for randomized controlled trials. 2017.

24. Borenstein M, Hedges L, Higgins J, Rothstein H. Comprehensive metaanalysis version 2. Englewood, NJ: Biostat. 2005;104

25. Higgins JP, Thompson SG, Deeks JJ, Altman DG. Measuring inconsistency in meta-analyses. BMJ. 2003;327:557-60.

26. Habtewold TD, Islam MA, Sharew NT, Mohammed SH, Birhanu MM, Tegegne BS. SystEmatic review and meta-aNAlysis of infanT and young child feeding Practices (ENAT-P) in Ethiopia: protocol. BMJ Open. 2017;7:e017437.

27. Luo M, Tan H, Zhou Q, Wang S, Cai C, Guo Y, et al. Realizing the metaanalysis of single rate in R software. J Evid Based Med. 2013;13:181-4.

28. Lewis S, Clarke M. Forest plots: trying to see the wood and the trees. BMJ. 2001;322:1479-80.
29. Ogilvie D, Fayter D, Petticrew M, Sowden A, Thomas S, Whitehead M, et al. The harvest plot: a method for synthesising evidence about the differential effects of interventions. BMC Med Res Methodol. 2008:8:8.

30. Tsumoto S, Hirano S. Formal analysis of leave-one-out methods based on decremental sampling scheme. 2014;2:371-8.

31. Gonzales DA, Norsworthy KJ, Kern SJ, Banks S, Sieving PC, Star RA, et al. A meta-analysis of $\mathrm{N}$-acetylcysteine in contrast-induced nephrotoxicity: unsupervised clustering to resolve heterogeneity. BMC Med. 2007:5:32.
Ready to submit your research? Choose BMC and benefit from:

- fast, convenient online submission

- thorough peer review by experienced researchers in your field

- rapid publication on acceptance

- support for research data, including large and complex data types

- gold Open Access which fosters wider collaboration and increased citations

- maximum visibility for your research: over $100 \mathrm{M}$ website views per year

At BMC, research is always in progress.

Learn more biomedcentral.com/submissions 